\title{
Tunable wave packet transport in branched periodic lattices with time-dependent external field
}

\author{
Jambul Yusupov \\ Turin Polytechnic University in Tashkent, 17. Niyazov Str., 100095, Tashkent, Uzbekistan \\ j.yusupov@polito.uz
}

PACS 03.65.Aa, 37.10.Jk, 05.60.Gg

DOI 10.17586/2220-8054-2017-8-1-42-47

\begin{abstract}
We study the dynamics of a Gaussian wave packet in a branched system of periodic lattices driven by an external time-dependent field. Motivated by recent numerical experiments with the manipulation of wave packet transmission in quantum star graph, we provide further discussion based on some achievements obtained from one-dimensional tight-binding models with arbitrary external time-dependent fields and on numerical calculations.
\end{abstract}

Keywords: quantum graph, periodically-driven system, directed transport, wave packet dynamics.

Received: 12 August 2016

Revised: 3 September 2016

\section{Introduction}

Quantum particle dynamics in discrete and branched structures is of importance for many topics in condensed matter and nanoscale physics. Over past two decades a powerful method based on the use of so-called quantum graphs has been developed and has found numerous applications (see, e.g., [1,2] for review). This approach uses wave equations with the boundary conditions given on metric graphs. The latter are the set of bonds connected to each other at branching points often called vertices [1,3]. Such a method allows one to obtain complete solution of the problem for a given network topology and different boundary conditions. The main issue appearing in the study of particle transport in such systems is the problem of vertex scattering and/or transmission which plays crucial role in the manifestation of diffusive or ballistic transport in networks and discrete systems. Then, a key problem in the study of particle in branched systems becomes tunable transport where the tuning of particle motion from ballistic to diffusive or vice-versa is possible. Such a goal can be achieved by acting on the system with an external perturbation, such as electric or magnetic fields. For example, under the influence of static force, the system (called also tilted lattice in literature) may exhibit coherent oscillations, which is well-studied in tight-binding approach [4] and was experimentally confirmed by several groups [5-8]. The time-dependent tilted lattice allows one to direct the wave packet, which can be achieved by the modulation of the lattice amplitude [9-11] and/or by the modulation of an external field [12-16].

Recent progress made in the fabrication of quantum devices that can be modeled by branched and discrete structures motivate the study transport problems in quantum graphs [3]. Quantum particle dynamics in graphs have been studied in several works $[17,18]$. The concept of directed transport has been extended to quantum graphs and the possibility to manipulate the direction of the motion of Gaussian wave packet (GWP) through the bifurcation point of a quantum star graph has been shown in a recent numerical experiment [19].

In this paper, we study a GWP dynamics in branched periodic lattices under the influence of different external time-dependent fields. Branching is considered as being in the form of a metric star graph. In particular, we apply the results from the Ref. [16], where expression for trajectory of GWP center in the infinite chain driven by a time-dependent homogeneous field is obtained using the tight-binding model. We compare these results with the solution in the continuous model, which is considered in [19]. This paper is organized as follows. In the next section, we briefly recall the model for quantum star graph. In section 3, we discuss in detail wave packet dynamics in connected lattices with different modulated external fields. Finally, section 4 presents some concluding remarks.

\section{Model}

Our model is the same as that considered in Ref. [19], where directed transport in driven star graph has been studied. Each of the arms of the star graph is vested with a periodic lattice potential given by $V(x)=$ $V_{0} \cos (2 \pi x / d)$, and they are connected at a common (central) vertex. We assign the coordinate $x$ to each arm, which indicates the position along the arm; $x$ takes the value 0 at the common vertex. Moreover, each arm is 
driven by a time-dependent external field $F_{j}(t)=f_{j} \sin \left(\omega t+\phi_{j}\right)$, also being arm-dependent, and the parameters $f_{j}$ and $\phi_{j}$ are used to manipulate the GWP transition through the central vertex.

The time-dependent Schrödinger equation (in the units $\hbar=2 m=1$ ) for a star graph with $N$ arms is:

$$
i \frac{\partial}{\partial t} \Psi_{j}(x, t)=-\frac{\partial^{2}}{\partial x^{2}} \Psi_{j}(x, t)+\left[V(x)+F_{j}(t) x\right] \Psi_{j}(x, t), \quad \forall t, \quad 0 \leq x \leq L_{j}, \quad j=1, \ldots, N .
$$

The imposed conditions are:

$$
\left\{\begin{array}{l}
\Psi_{1}\left(L_{1}, t\right)=\Psi_{2}\left(L_{2}, t\right)=\ldots=\Psi_{N}\left(L_{N}, t\right)=0 \\
\Psi_{1}(0, t)=\Psi_{2}(0, t)=\ldots=\Psi_{N}(0, t) \\
\left.\sum_{j=1}^{N} \frac{\partial}{\partial x} \Psi_{j}(x, t)\right|_{x=0}=0
\end{array}\right.
$$

which imply Dirichlet boundary conditions at the non-connected edges in the form of continuity and current conservation at the vertex.

Referring to the results of the paper presented in Fig. 1, one can see dispersion of the packet after collision with vertex. This phenomenon is the starting point for the study of bifurcation at the graph vertex. Numerical method used in this work for the solution of the Schrödinger equation is the same as that in [19].
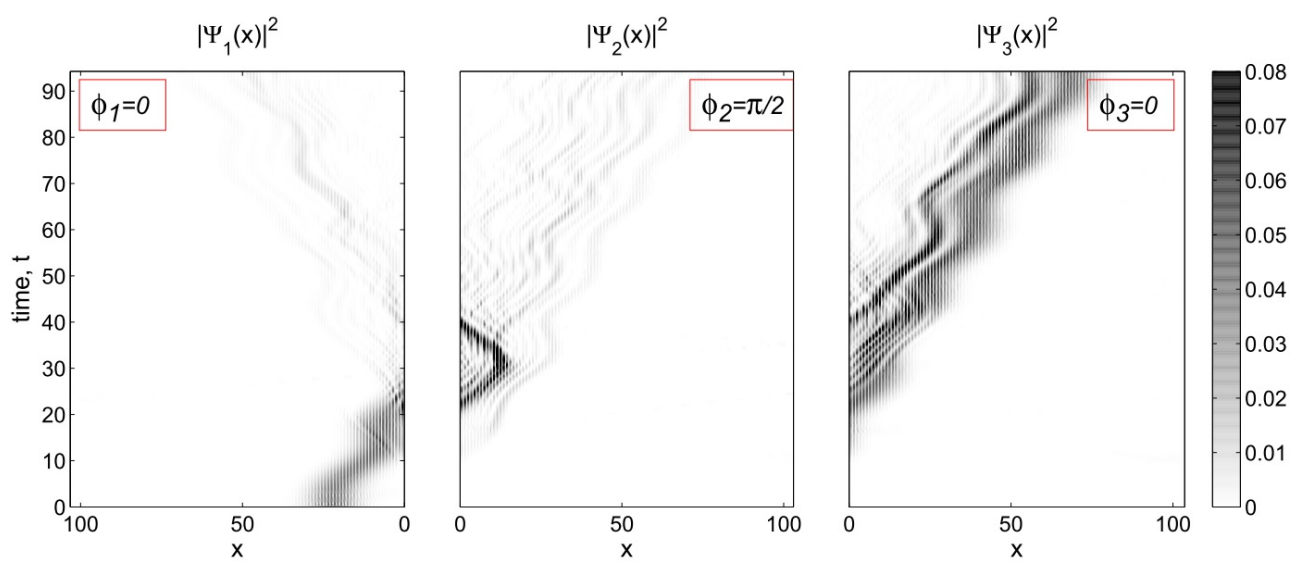

FIG. 1. Contour plot of the probability density for the three arm star graph, where the driving external field is given by $F_{j}(t)=f_{j} \sin \left(\omega t+\phi_{j}\right)$ with $f_{1}=-f_{2}=-f_{3}=\pi / 10, \omega=0.2$. The three columns correspond to the arms of the star graph. The $x$-coordinate of the first arm is reversed. (Fig. from [19])

\section{Wave packet dynamics}

The time evolution of Gaussian wave packet (GWP):

$$
\Psi\left(x, t=t_{0}\right)=\frac{1}{\sqrt{\sqrt{\pi} \sigma_{0}}} \exp \left(-\frac{\left(x-x_{0}\right)^{2}}{2 \sigma_{0}^{2}}+i k_{0} x\right),
$$

in a system driven by an arbitrary time-dependent external field was studied in [16] using the tight-binding approximation. According to this paper, in the case of a chain, momentum changes as:

$$
k=k_{0}-I\left(t, t_{0}\right) \text {, }
$$

where

$$
I\left(t, t_{0}\right)=\int_{t_{0}}^{t} F(\tau) d \tau
$$

for arbitrary $F(t)$. As we consider the time-dependence of the external field given as $F(t)=f \sin (\omega t+\phi)$, one obtains:

$$
I\left(t, t_{0}\right)=-\frac{f}{\omega}\left[\cos (\omega t+\phi)-\cos \left(\omega t_{0}+\phi\right)\right] .
$$


In the case of a wide wave packet, for the group velocity we have:

$$
v_{G}(t)=2 J \sin \left[k_{0}-I(t)\right],
$$

where $J$ is hopping amplitude. Consequently, the displacement is given by:

$$
D(t)=\int_{0}^{t} v_{G}(\tau) d \tau .
$$

Furthermore, this expression is used for the calculation of GWP trajectory, which is compared with the solution of the Schrödinger equation in continuous model.

\subsection{Trajectory of GWP center}

Without the loss of generality, we can consider the case of two bonds connected at one common vertex. Each bond is vested with periodic potential and driven by the modulated external field. For this system, we have no reflection at the common vertex (due to the Neumann boundary conditions), and when parameters of the fields are chosen as $f_{1}=-f_{2}=\pi / 10, \phi_{1}=\phi_{2}=0, \omega=0.2$ one is faced with the chain problem. The signs of parameters $f_{1}$ and $f_{2}$ are chosen such that the field points globally in one direction. According to the above derivations, one can evaluate the trajectory of the GWP center and compare them with the solution of the Schrödinger equation (Fig. 2).
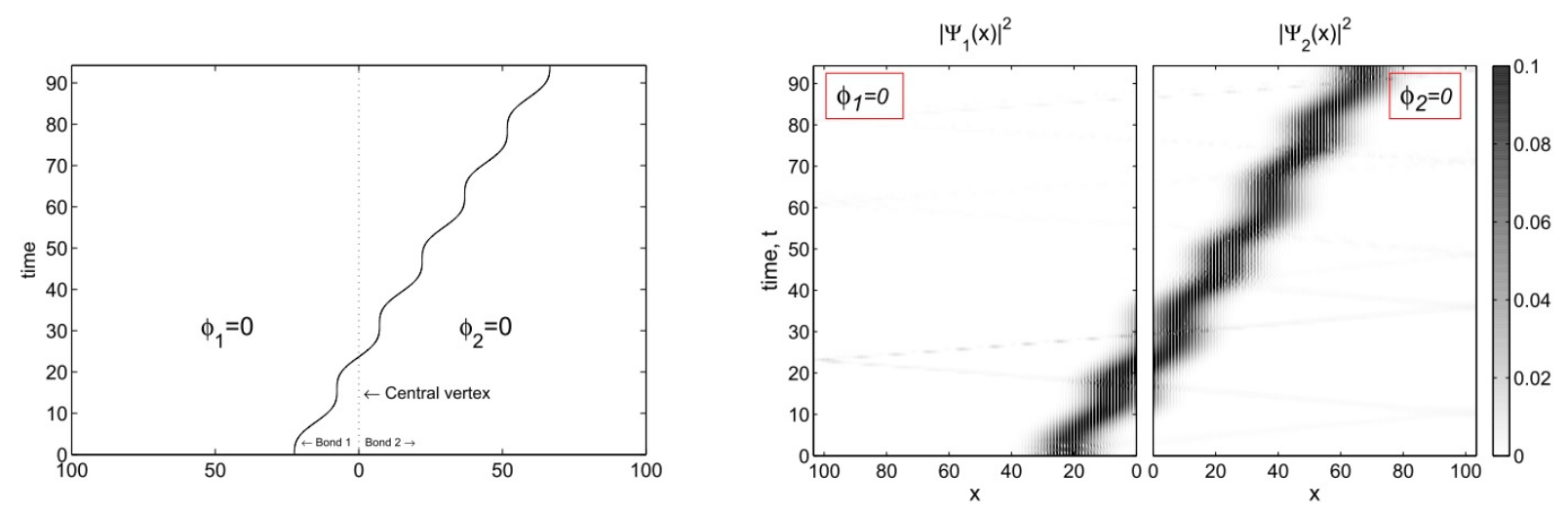

FIG. 2. A GWP center's trajectory (left) and the solution of the Schrödinger equation for two connected bonds (right) for $F_{j}(t)=f_{j} \sin \left(\omega t+\phi_{j}\right)$ with $f_{1}=-f_{2}=\pi / 10, \phi_{1}=\phi_{2}=0$, $\omega=0.2$ and $V_{0}=16.7875$ (corresponding to the hopping amplitude $J=1$ in the tight-binding model)

The situation changes when we introduce phases $\phi_{j}$ being different for each bond. Starting to move from one bond, a wave packet reaches the common vertex. After collision, the particle falls into another medium with a different external field. In our case, the difference is in the phase $\phi$. Each time when reaching a common boundary, the wave packet starts a "new" trajectory in a corresponding external field with "new initial" momentum $k$, which makes the dynamics strongly dependent on the initial state of the GWP. Comparison of results for the two approaches is presented in Fig. 3 for the same parameters as in Fig. 2 with the only difference in $\phi_{2}=\pi / 2$.

As one can see from these results, we still have dissipation of the wave packet at the vertex. The same dynamics are seen from the first and second bonds in Fig. 1. The difference is on the small fraction of the packet, which appears from reflection on the common vertex. Thus, for a system of connected lattices, the expression for the GWP center's trajectory is not valid. This can be explained by the localization of wave packets on the lattice sites (Fig. 4).

Now, based on this idea, we can compute trajectories for the localized wave packets separately. Of course, this does not mean that a single wave packet localized in one site behaves in this way. We again note that we consider here a set of localized GWPs enveloped in a wide Gaussian wave packet. In Fig. 5, trajectories of localized wave packets are presented. The gray gradient in the left figure introduced in order to depict wave packets with different amplitudes; darker colors correspond to larger amplitudes. These results can be compared with the solution of the Schrödinger equation for two connected bonds. 

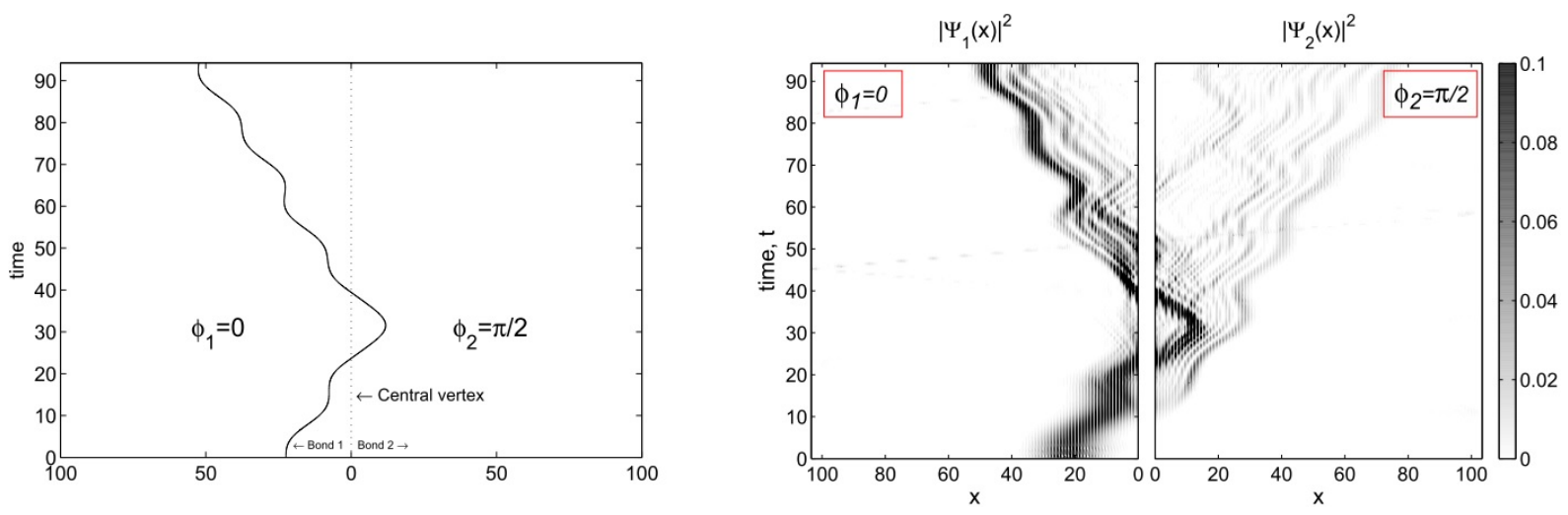

FIG. 3. A GWP center's trajectory (left) and the solution of the Schrödinger equation for two connected bonds (right) for the same parameters as in Fig. 2 with the only difference in $\phi_{2}=\pi / 2$.

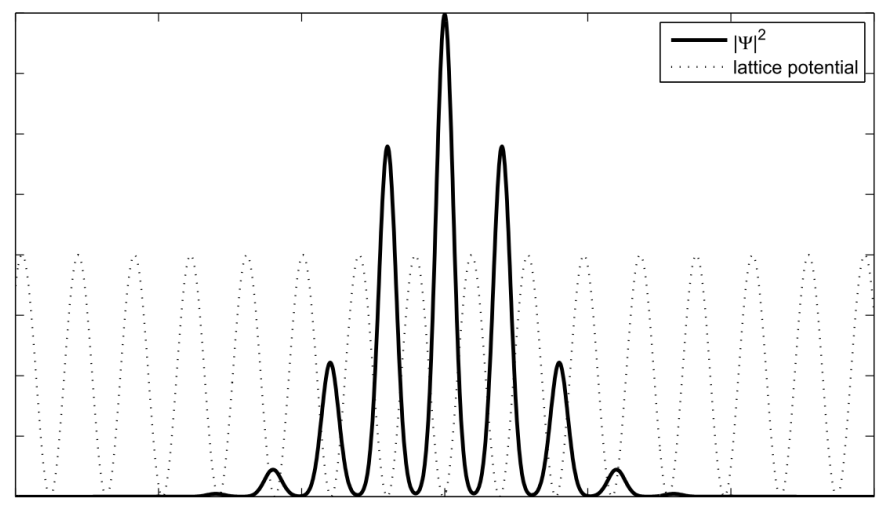

FIG. 4. Sketch of the profile of GWP in the lattice given by a cosine potential
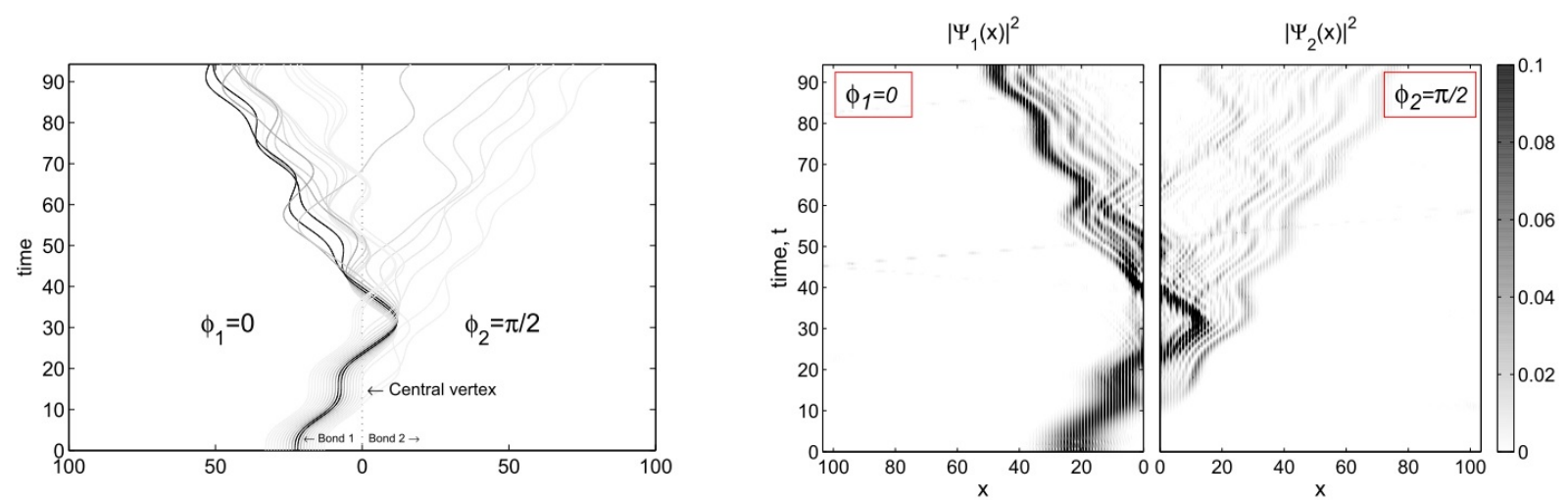

FIG. 5. Trajectories of localized wave packets (left) compared with the solution of the Schrödinger equation for two connected bonds (right). Gray gradient in the left figure; darker colors correspond to larger amplitudes

\subsection{Manipulating GWP dynamics}

A naive trial to bring to stop a GWP in the central vertex can be done in the following way. According to Eq. (6), in order to bring to a stop a wave packet, one needs in general:

$$
k_{0}+\frac{f}{\omega}\left(\cos (\omega t+\phi)-\cos \left(\omega t_{0}+\phi\right)\right)= \pm \pi n, \quad n=0,1, \ldots
$$

Therefore, one of the solutions is $k_{0}= \pm \pi n$ and $f=0$ (or $\omega \gg f$ ) at the same time. In this case, $k_{0}$ is the initial momentum to the second bond and consequently $f_{2}=0$. Selecting $k_{0}= \pm \pi n$ means selecting proper 
initial position $x_{0}$. So, for the initial momentum $k_{0}=0$ in the first bond, we can obtain periodically this value in each time $t_{n}=2 \pi n / \omega$. Then, in order the target position to be 0 (to stop at the common vertex), one can compute $x_{0}=D\left(t_{n}\right)$. Thus, direct computations for parameters $f_{1}=\pi / 10, f_{2}=0, \omega=0.2$ result in Fig. 6 , where $x_{0}=29.2166$.

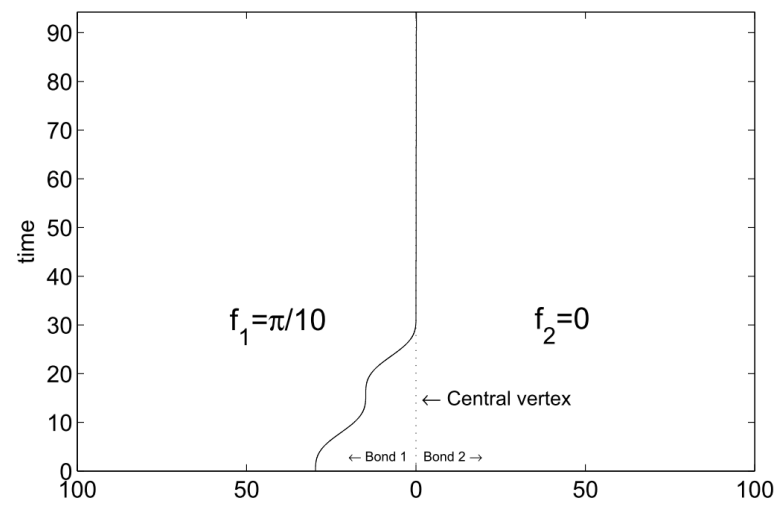

FIG. 6. Vanishing displacement of GWP center: $f_{1}=\pi / 10, f_{2}=0, \omega=0.2$ and $x_{0}=29.2166$.

Another possibility to block transition can be achieved by choosing parameters as $f_{1}=-f_{2}=\pi / 10, \phi_{1}=0$, $\phi_{2}=-\pi, \omega=0.2$ and $x_{0}=29.6567$ is the initial position of GWP's center, which correspond to having $k=0$ on the common vertex. In such a case, one expects the trajectory shown in Fig. 7.

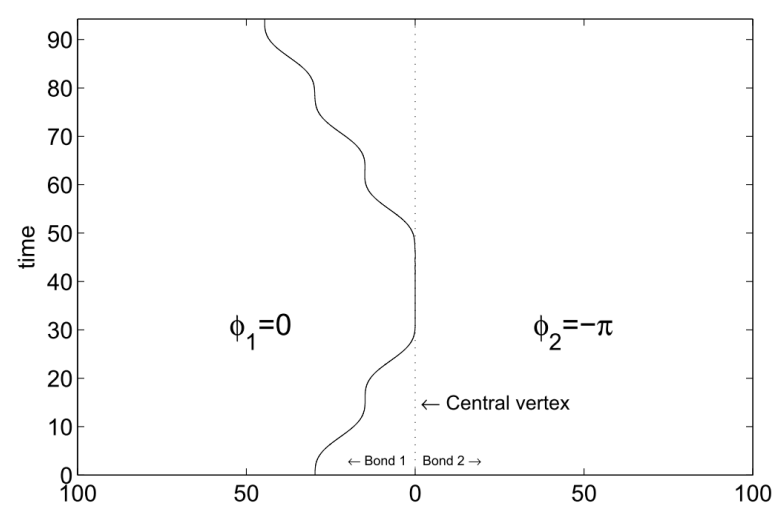

FIG. 7. Total reflection of a GWP in the central vertex: $f_{1}=-f_{2}=\pi / 10, \phi_{1}=0, \phi_{2}=-\pi$, $\omega=0.2$ and $x_{0}=29.2166$.

At first glance, it seems easy that we found parameters to stop or totally reflect GWP at the vertex. However, solving the Schrödinger equation for these parameters results in different dynamics (see Fig. 8).

Thus, in order to be able to control GWP dynamics, one needs initialize localized wave packets with different precalculated group velocities. Despite the fact that this set up is experimentally unrealizable, theoretically it is possible to control GWP in this way.

\section{Conclusion}

In this paper, we have studied Gaussian wave packet dynamics in connected lattices under influence of different external time-dependent fields. It is shown that the dispersion of the wave packet at the bifurcation point is not only caused by reflection and transmission rules but also by the localization of the wave packet in the lattice sites. It was determined that the trajectory of a GWP should be computed for localized wave packets in order to obtain a complete picture of the dynamics and its calculation for branched lattices with different modulated fields is not valid. Even if this paper dealt with two connected lattices, the concept also holds true for many bond star graphs.

According to this approach, it is theoretically possible to control transmission or reflection of the GWP by selecting proper initial conditions (for example by assigning proper initial momentum to the each localized wave packet, which would be practically impossible to do) and parameters of the field, which will be studied in future works. 

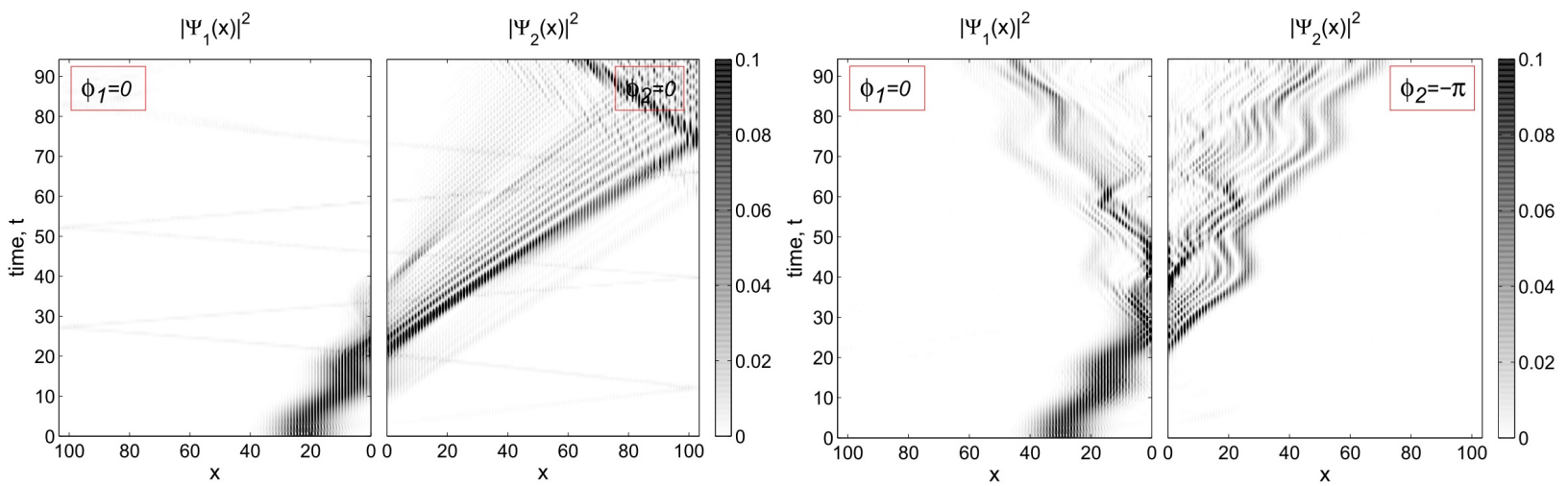

FIG. 8. Solution of the Schrödinger equation for cases considered in Fig. 6 (left) and Fig. 7 (right).

\section{Acknowledgements}

This work is supported by a grant of the Committee for Coordination of the development of Science and Technology of Uzbekistan (Grant No F-2-003).

\section{References}

[1] Gnutzmann S., Smilansky U. Quantum graphs: Applications to quantum chaos and universal spectral statistics. Adv. Phys., 2006, 55 (5-6), P. 527-625.

[2] Exner P., Kovařík H. Quantum waveguides. Springer, 2015.

[3] Kottos T., Smilansky U. Periodic orbit theory and spectral statistics for quantum graphs. Ann. Phys., 1999,274 (1), P. $76-124$.

[4] Hartmann T., Keck F., Korsch H.J., Mossmann S. Dynamics of bloch oscillations. New J. Phys., $2004,6,2$.

[5] Dekorsy T., Ott R., Kurz H., Köhler K. Bloch oscillations at room temperature. Phys. Rev. B, 1995, 51, P. $17275-17278$.

[6] Ben Dahan M., Peik E., Reichel J., et al. Bloch oscillations of atoms in an optical potential. Phys. Rev. Lett., 1996,76, P. $4508-4511$.

[7] Haller E., Hart R., et al. Inducing transport in a dissipation-free lattice with super bloch oscillations. Phys. Rev. Lett., 2010 , 104, P. 200403.

[8] Lehtinen J.S., Zakharov K., Arutyunov K.Yu. Coulomb blockade and bloch oscillations in super-conducting Ti nanowires. Phys. Rev. Lett., 2012, 109, P. 187001.

[9] Alberti A., Ferrari G., et al. Atomic wave packets in amplitude- modulated vertical optical lattices. New Journal of Physics, 2010, 12, P. 065037.

[10] Mülken O., Bauer M. Directed excitation transfer in vibrating chains by external fields. Phys. Rev. E, 2011,83 , P. 061123.

[11] Thommen Q., Garreau J.C., Zehnlé V. Quantum motor: Directed wave- packet motion in an optical lattice. Phys. Rev. A, 2011, 84, P. 043403.

[12] Thommen Q., Garreau J.C., Zehnlé V. Theoretical analysis of quantum dynamics in one-dimensional lattices: Wannier-stark description. Phys. Rev. A, 2002, 65, P. 053406.

[13] Thommen Q., Garreau J.C., Zehnlé V. Atomic motion in tilted optical lat- tices: an analytical approach. J. Opt. B, 2004, 6 (7), P. 301.

[14] Arlinghaus S., Holthaus M. Controlled wave-packet manipulation with driven optical lattices. Phys. Rev. A, 2011,84 , P. 063617.

[15] Creffield C.E., Sols F. Directed transport in driven optical lattices by gauge generation. Phys. Rev. A, 2011, 84, P. 023630.

[16] Hu W.H., Jin L., Song Z. Dynamics of one-dimensional tight-binding models with arbitrary time-dependent external homogeneous fields. Quantum Inf. Process., 2013, 12 (11), P. 3569-3585.

[17] Matrasulov D.U., Yusupov J.R., Sabirov K.K., Sobirov Z.A. Time-dependent quantum graph. Nanosystems: Physics, Chemistry, Mathematics, 2015, 6 (2), P. 173-181.

[18] Yusupov J., Eshniyozov V., Karpova O., Saidov D.Sh. Kicked particle dynamics in quantum graphs. Nanosystems: Physics, Chemistry, Mathematics, 2015, 6 (6), P. 773-778.

[19] Yusupov J., Dolgushev M., Blumen A., Mülken O. Directed transport in quantum star graphs. Quantum Information Processing, 2016, 15 (4), P. 1765-1777. 